\title{
Metabolic profiling of hepatitis B virus-related hepatocellular carcinoma with diverse differentiation grades
}

\author{
LEI ZHANG ${ }^{1,2^{*}}$, YA HUANG $^{3 *}$, MINGJIAN LIAN $^{4 *}$, ZHIJUAN FAN $^{5}$, YAQIONG TIAN $^{5}$, YUFAN WANG $^{5}$, \\ HUA KANG ${ }^{5}$, SHUANG LIU ${ }^{4}$, SHUYE LIU ${ }^{5}$, TONG LI ${ }^{5}$ and ZHONGQIANG SHAN ${ }^{2}$ \\ ${ }^{1}$ Tianjin Key Laboratory of Artificial Cell, Artificial Cell Engineering Technology Research Center of Public Health Ministry, \\ Tianjin Third Central Hospital, Tianjin 300170; ${ }^{2}$ Chemical Engineering Institute, Tianjin University, Tianjin 300072; \\ ${ }^{3}$ Clinical Laboratory Department, Chongqing City First People's Hospital of Wanzhou, Chongqing 404040; \\ ${ }^{4}$ Clinical Laboratory Department, Third Central Clinical College, Tianjin Medical University, Tianjin 300070; \\ ${ }^{5}$ Tianjin Key Laboratory of Artificial Cell, Clinical Laboratory Department, \\ Tianjin Third Central Hospital, Tianjin 300170, P.R. China
}

Received March 14, 2016; Accepted September 9, 2016

DOI: $10.3892 / 01.2017 .5596$

\begin{abstract}
The most effective diagnostic tool for the majority of hepatocellular carcinoma (HCC) patients is determining the differentiation grade of their tumors. However liver biopsies, which are currently the most effective way of determining tumor differentiation grade, have several limitations. The present study was designed to select serum characteristic metabolites that correlate with the differentiation grades of hepatitis $\mathrm{B}$ virus (HBV)-related HCC, and so could be used in the clinic as a non-invasive method of differentiating patients with different grades of HCC. A total of 58 patients with HBV-related HCC were included in the present study, and divided into three groups according to their tumor differentiation grade. A further 20 patients with HBV-related liver cirrhosis and 19 healthy volunteers were enrolled. Ultra-performance liquid chromatography-mass spectrometry was used to analyze endogenous metabolites. Multivariate statistical analysis was used to examine the data using MZmine 2.0 software. The 14 metabolites that were highly correlated with specific differentiation grades of HCC were then selected for additional study. Receiver operator characteristic curve analysis was used to
\end{abstract}

Correspondence to: Dr Shuye Liu, Tianjin Key Laboratory of Artificial Cell, Clinical Laboratory Department, Tianjin Third Central Hospital, 83 Jintang Road, Hedong, Tianjin 300170, P.R. China

E-mail: rikkichang12345@163.com

Professor Zhongqiang Shan, Chemical Engineering Institute, Tianjin University, 72 Weijin Road, Nankai, Tianjin 300072, P.R. China

E-mail: shanzq@sohu.com

*Contributed equally

Key words: hepatocellular carcinoma metabolomics, characteristic metabolites, ultra performance liquid chromatography-mass spectrometry evaluate their clinical value. In total, 5 metabolites were finally identified, including lysophosphatidylcholine (16:0), oleamide, monoglyceride (0:0/15:0/0:0), lysophosphatidylcholine (18:0) and lysophosphatidylcholine [22:5(7Z,10Z,13Z,16Z,19Z)]. All these metabolites exhibited an excellent ability to distinguish different types of HCC with various differentiation grades and the area under the curve of these metabolites was up to 0.942 , showing promising clinical value.

\section{Introduction}

Hepatocellular carcinoma (HCC) is the fifth most common cancer and the third leading cause of cancer deaths worldwide with a $<7 \%$ five-year survival rate (1-3). Globally, infection by the hepatitis $\mathrm{B}$ virus (HBV) is the most prevalent cause of HCC. The prognosis of HCC can be influenced by several clinicopathological factors, such as liver function, number of nodules, tumor size, vascular invasion and differentiation grade (4-6). Among these, tumor differentiation grade is the most significant factor in deciding on the type of therapy that HCC patients should receive. The most widely used method to assess differentiation grade are the Edmondson-Steiner criteria (7). However, this is currently achieved using liver biopsy, which may increase the risk of HCC further developing, for example, exposing patients to the risk of tumor cell-seeding (8). In addition, needle biopsies have a significant false-negative rate for the diagnosis of HCC due to sampling errors (9). Furthermore, determination of the Edmondson grades is likely to be affected by subjective factors and therefore, additional studies that focus on the exploration of non-invasive prediction of differentiation are required.

Recent advances in analytical chemistry have resulted in metabolomics becoming an important way of understanding disease mechanisms and identifying candidate biomarkers. Metabolomics is defined as the comprehensive quantitative and qualitative analysis of all metabolites in cells, tissues, or biofluids in response to biological interventions or environmental factors $(10,11)$. An increasing number of studies focus 
on the pathogenesis and biomarkers of HCC using metabolomics methodology. Thus metabolomics may provide a way to select characteristic metabolites that can distinguish HCC patients with diverse differentiation grades without having to carry out a biopsy.

In the current study, an ultra performance liquid chromatography and linear trap quadrupole (UPLC-LTQ)-Orbitrap XL mass spectrometry (MS) analytical platform was used to analyze the serum metabolic profiling of HBV-related HCC patients with diverse differentiation grades. Following selection and identification of the metabolites, their clinical value was assessed by receiver operator characteristic curve (ROC) analysis and area under the curve (AUC) analysis.

\section{Materials and methods}

Chemicals and instruments. All solvents used in the present study were high performance liquid chromatography (HPLC) grade without modification. Formic acid and acetonitrile were obtained from Merck (Merck Millipore, Darmstadt, Germany). Distilled water was produced using a Milli-Q Reagent Water System (EMD Millipore, Billerica, MA, United States). Standard preparations of Lysophosphatidylcholine (LysoPC) (16:0) and LysoPC (18:0) were purchased from Sigma-Aldrich (St. Louis, MO, USA). Calibration standards [caffeine, Ultramark 1621 and methionine-arginine-phenylalanine-alanine (MRFA)] were provided by Thermo Fisher Scientific Inc. (Waltham MA, United States). UPLC was performed using an Accela system (Thermo Fisher Scientific Inc. Waltham, MA, United States). MS was performed with a LTQ Orbitrap XL hybrid mass spectrometer (Thermo Fisher Scientific Inc.).

Enrolled population and sample collection. A total of 58 HBV-related HCC patients were included in the current study. All samples were obtained between October 2013 and March 2015 from inpatients and outpatients at the Tianjin Third Central Hospital, Tianjin, China. HCC was diagnosed in accordance with clinical practice guidelines proposed in 2012 (12), and the diagnostic results were confirmed by histopathological examination. The patients were divided into three groups according to the Edmondson-Steiner criteria: Group A included 21 patients with high-grade HCC (Edmondson-Steiner I), group B included 23 patients with middle-grade HCC (Edmondson-Steiner II), and group C included 14 patients with low-grade HCC (Edmondson-Steiner III/IV). The pathological images of HCC tissue are presented in Fig. 1. A further 20 patients were diagnosed with HBV-related liver cirrhosis (LC), and were all classified as Child-Pugh A grade (13). These patients were put into a different group, called Group LC. All of the patients within the current study were confirmed to have no secondary liver cancer, other systemic tumors, diabetes or other metabolic diseases. A control group was also included, consisting of 19 healthy volunteers, all were confirmed to have normal liver function, no viral hepatitis, no alcohol or nonalcohol fatty liver, no metabolic disease or other complications. The demographic and clinical characteristics of all participants are presented in Table I.

None of the participants were on any medical treatment for the 4 weeks prior to sample collection. Fasting blood samples were collected and centrifuged at 2,000 x $g$ for $30 \mathrm{~min}$. The sera were separated, aliquoted and stored at $-80^{\circ} \mathrm{C}$.

The study protocol was approved by the Hospital Ethics Committee of Tianjin Third Central Hospital and adhered to the tenets of the Declaration of Helsinki. All participants voluntarily joined this study and provided their informed consent prior to commencing any study procedures.

Sample preparation. Prior to UPLC/MS analysis, serum samples were thawed at room temperature and an aliquot of $100 \mathrm{ml}$ of serum sample was mixed with $300 \mathrm{ml}$ methanol. The mixture was vibrated for 30 s and then left to stand for $45 \mathrm{~min}$ at room temperature. The mixture was then centrifuged at $4^{\circ} \mathrm{C}$ at $10,000 \times \mathrm{g}$ for $30 \mathrm{~min}$. The supernatant was filtered using a $0.22 \mu \mathrm{m}$ membrane (Merck Millipore, Darmstadt, Germany).

Sample analysis. Chromatography was performed using an Accela system (Thermo Fisher Scientific Inc.) equipped with a binary solvent delivery manager and a sample manager. The analytical column was a Thermo Hypersil GOLD (2.1 $\left.\mathrm{mm} \mathrm{xID}_{50} \mathrm{~mm}, 1.9 \mu \mathrm{m}\right) \mathrm{C} 18$ reverse phase column. The injected volume was $10 \mu \mathrm{l}$ and the flow rate was maintained at $200 \mu \mathrm{l} / \mathrm{min}$. The temperatures of the sample manager and column oven were set at $4^{\circ} \mathrm{C}$ and $20^{\circ} \mathrm{C}$, respectively. For UPLC analysis, the mobile phase consisted of $0.1 \%$ formic acid aqueous solution (phase A) and $0.1 \%$ formic acid acetonitrile solution (phase B) (Merck Millipore). Chromatographic separation was performed within 21 min per sample: i) $95 \%$ phase A and 5\% phase B were held for 2.5 min initially; ii) Phase B was gradually escalated to $95 \%$ in the following $7 \mathrm{~min}$; iii) Phase B was maintained at $95 \%$ for 3 min and then gradually reduced to $5 \%$ in the following $6 \mathrm{~min}$; iv) $5 \%$ phase B was held for $2.5 \mathrm{~min}$ to balance the analytical column.

MS was performed in positive mode. The following parameters were employed: Ion spray voltage, $4.5 \mathrm{kV}$; capillary voltage, $30 \mathrm{~V}$; cone voltage, $150 \mathrm{~V}$; desolvation temperature, $350^{\circ} \mathrm{C}$; sheath gas flow rate of 30 arb and assistant gas flow rate of 5 arb (99.999\% nitrogen). Data were collected in centroid mode and the mass-to-charge ratio $(\mathrm{m} / \mathrm{z})$ range was set at 50-1000. MS resolution was 100,000 full width at half maximum (FWHM) and calibration standards were used, including caffeine, Ultramark 1621 and MRFA (Thermo Fisher Scientific Inc.). MS/MS analysis was performed by using collision-induced dissociation at $35 \%$ normalization collision energy and the collision gas was $99.999 \%$ helium.

Statistical analysis. MZmine 2.0 software (14) was used for peak detection, alignment and normalization. The filter conditions were: Each chromatography peak signal-to-noise ratio $>30$, the retention time tolerance at $\pm 0.1 \mathrm{~min}$ and the $\mathrm{m} / \mathrm{z}$ tolerance at $\pm 0.01 \mathrm{Da}$. SIMCA-P+ ver. 12.0.1.0 software (Umetrics, Malmö, Sweden) was used to establish the principal component analysis (PCA) and orthogonal partial least squares discriminant analysis (OPLS-DA) model of all the samples and the result was checked by cross validation described previously $(15,16)$. Preliminary selection of characteristic metabolites was accomplished using the corresponding variable influence on projection (VIP) value, confidence interval and coefficient plot generated by the OPLS-DA model. SPSS ver. 17.0 software (SPSS Inc., 
Table I. Clinical parameters for HCC, LC, and control groups.

\begin{tabular}{lccccc}
\hline & \multicolumn{5}{c}{ HCC group (n=58) } \\
Parameters & Group A (n=21) & Group B (n=23) & Group C (n=14) & Group LC (n=20) & $\begin{array}{c}\text { Control group } \\
(\mathrm{n}=19)\end{array}$ \\
\hline Male/female & $14 / 7$ & $17 / 6$ & $11 / 3$ & $14 / 6$ & $12 / 7$ \\
Age & $56.4 \pm 5.4$ & $56.7 \pm 6.2$ & $55.3 \pm 5.8$ & $56.3 \pm 5.3$ & $57.0 \pm 6.1$ \\
ALB (g/l) & $40.30(37.90-47.30)$ & $40.80(39.55-44.85)$ & $40.55(39.17-43.57)$ & $41.23(37.54-42.57)$ & $47.70(45.4-49.40)$ \\
ALT (U/l) & $33.00(19.00-60.13)$ & $39.00(22.00-79.50)$ & $47.00(26.50-78.50)$ & $29(19.00-40.00)$ & $18.00(15.00-21.00)$ \\
AST (U/l) & $34.00(27.00-57.50)$ & $34.00(22.00-71.50)$ & $39.00(16.75-75.84)$ & $27.5(18.00-43.25)$ & $20.00(17.00-24.00)$ \\
GGT (U/1) & $56.00(32.00-78.00)$ & $71.00(45.50-160.00)$ & $103.50(58.75-147.50)$ & $35(19.00-53.00)$ & $16.00(13.00-26.00)$ \\
TBA $(\mu \mathrm{mol} / \mathrm{l})$ & $8.70(4.10-11.87)$ & $8.80(4.15-22.14)$ & $9.00(6.55-16.02)$ & $0.86(0.50-1.87)$ & $1.90(1.200-2.400)$ \\
AFP $(\mathrm{ng} / \mathrm{ml})$ & $145.29(3.96-897.29)$ & $156.45(2.34-1103.23)$ & $152.97(12.14-1210.24)$ & $3.19(1.21-9.85)$ & $2.71(2.11-3.38)$ \\
\hline
\end{tabular}

HCC, hepatocellular carcinoma; LC, liver cirrhosis; ALB, albumin; ALT, alanine aminotransferase; AST, aspartate aminotransferase; GGT, gamma-glutamyl transpeptidase; TBA, total bile acid; AFP, alpha-fetoprotein. All data are presented as median (range), except age and gender.
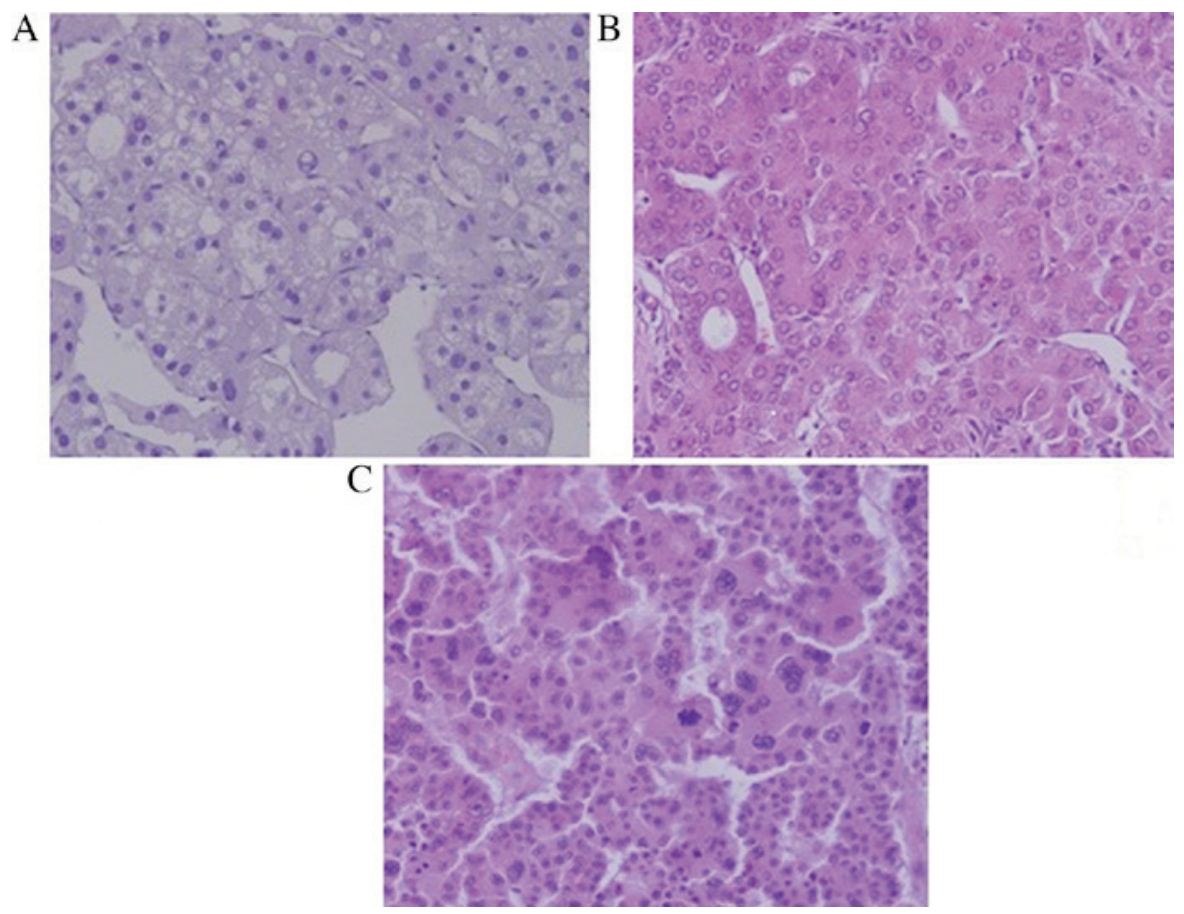

Figure 1. Pathological image of hepatocellular carcinoma (HCC) tissues (hematoxylin-eosin staining, magnification, $\mathrm{x} 400$ ). (A) High-grade differentiated HCC, (B) middle-grade differentiated HCC, and (C) low-grade differentiated HCC.

Chicago, IL United States) was used to evaluate the statistical significance of differences of the variances among diverse groups. The ROC curves were generated and the corresponding AUC was calculated. $\mathrm{P}<0.05$ was considered to indicate a statistically significant difference.

Identification of the characteristic metabolites. Some characteristic ions were identified by comparing the secondary mass spectrum and retention index with authentic reference standards. Due to the high resolution of the Orbitrap XL MS (100,000 FWHM), other characteristic metabolites were identified by checking the accurate $\mathrm{m} / \mathrm{z}$ value against the Human Metabolome DataBase (http://hmdb.ca). Matching substances that had $\mathrm{m} / \mathrm{z}$ deviations $<0.01$ were considered for further identification when the ionization modes were the same as those used in the HMDB database. After MS/MS scanning, the secondary mass spectrometer (MS2) spectra of characteristic ions were obtained and compared with theoretical fragments from preliminary results. This established that the MS2 m/z deviation was $<0.2$, the top three peaks matched and that there was $\geq 80 \%$ match between the preliminary and secondary mass spectra. The theoretical fragments were derived using Mass Frontier 6.0 software (Thermo Fisher Scientific Inc.).

\section{Results}

Metabolic profile. The total ion chromatograms of a single sample from each patient group acquired by the UPLC-MS 


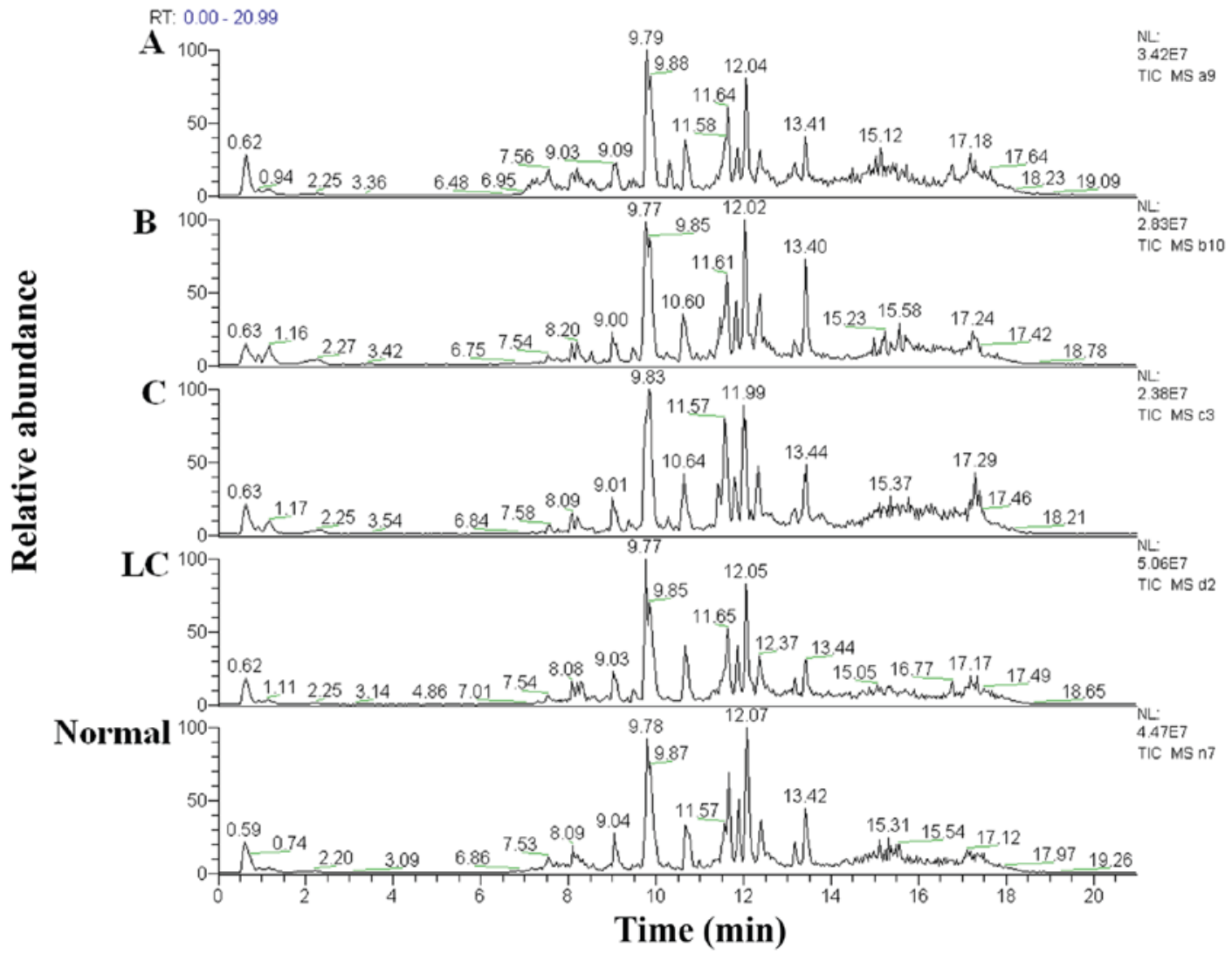

Figure 2. Total ion chromatogram of a single sample from each group was chosen randomly. (A) High-grade differentiated hepatocellular carcinoma (HCC), (B) middle-grade, (C) low-grade, (LC): Liver cirrhosis, (healthy): Healthy volunteers. The RT, retention time was the same for all groups.
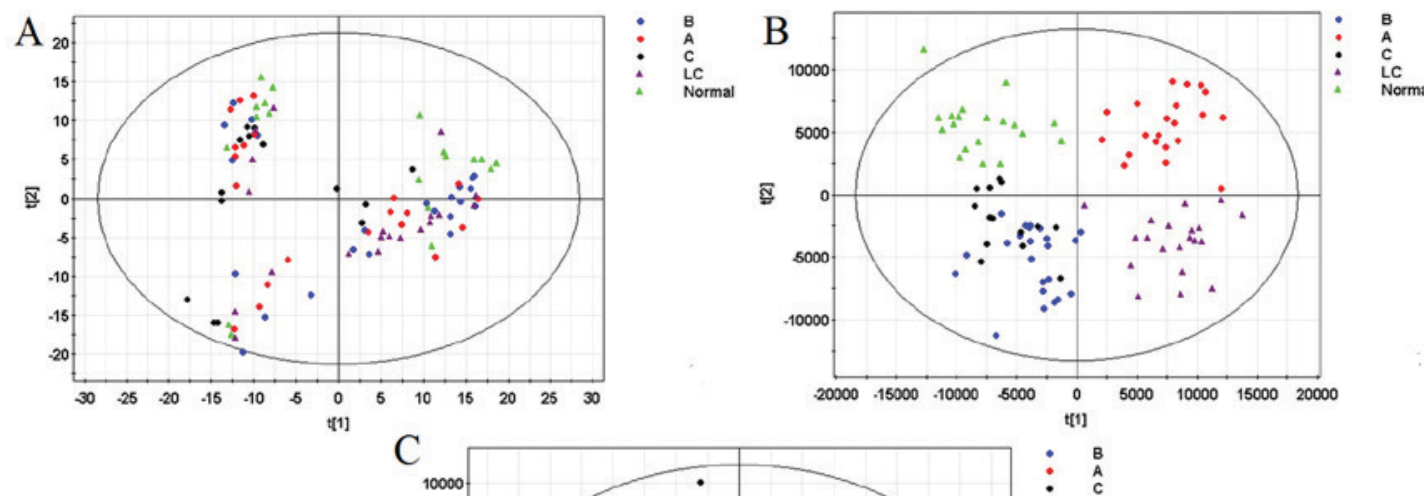

$\mathrm{C}$

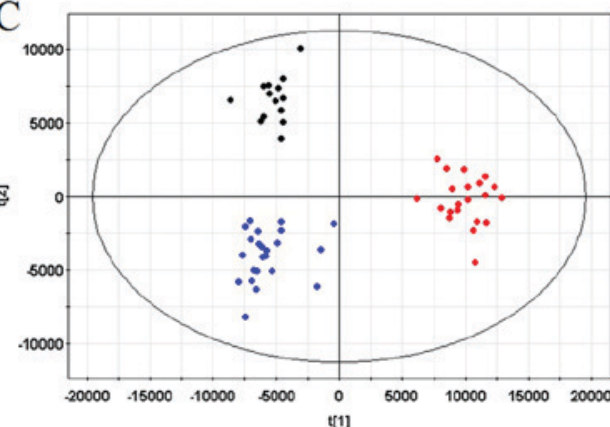

Figure 3. The ability of metabolic profiling to distinguish hepatocellular carcinoma (HCC) patients with diverse differentiation grades. (A) Principal component analysis (PCA) model with all samples, (B) orthogonal partial least squares discriminant analysis (OPLS-DA) model with all samples, and (C) OPLS-DA model with samples only from HCC patients.

platform are presented in Fig. 2. Using the MZmine ver. 2.0 software, this pre-treatment sought out 950 integral peaks following extraction ion chromatography detection in all samples. Following pre-treatment, a PCA model was established ( $\mathrm{R} 2 \mathrm{X}=44.7 \%, \mathrm{Q} 2=19.4 \%)$, as well as an OPLS-DA model ( $\mathrm{R} 2 \mathrm{X}=74.5 \%, \mathrm{R} 2 \mathrm{Y}=82.5 \%$, $\mathrm{Q} 2=49.6 \%$ ), using data 
Table II. Metabolite identification.

\begin{tabular}{lcccc}
\hline \multirow{2}{*}{ Metabolite } & & \multicolumn{3}{c}{ Content $^{\mathrm{c}}$} \\
\cline { 3 - 5 } & Adduct $^{\mathrm{b}}$ & $\mathrm{B} / \mathrm{A}$ & $\mathrm{C} / \mathrm{B}$ & C/A \\
\hline LysoPC $(16: 0)^{\mathrm{a}}$ & {$[\mathrm{M}+\mathrm{H}]^{+}$} & Down $(\mathrm{P}=0.01)$ & & Down $(\mathrm{P}=0.001)$ \\
Oleamide & {$\left[\mathrm{M}+\mathrm{Na}^{+}\right.$} & Down $(\mathrm{P}=0.001)$ & Down $(\mathrm{P}=0.001)$ & Down $(\mathrm{P}=0.001)$ \\
MG $(0: 0 / 15: 0 / 0: 0)$ & {$\left[\mathrm{M}+\mathrm{NH}_{4}\right]^{+}$} & & Down $(\mathrm{P}=0.003)$ & Down $(\mathrm{P}=0.001)$ \\
LysoPC $(18: 0)$ & {$[\mathrm{M}+\mathrm{Na}]^{+}$} & & Down $(\mathrm{P}=0.001)$ & Down $(\mathrm{P}=0.001)$ \\
LysoPC $[22: 5(7 \mathrm{Z}, 10 \mathrm{Z}, 13 \mathrm{Z}, 16 \mathrm{Z}, 19 \mathrm{Z})]$ & {$[\mathrm{M}+\mathrm{H}]^{+}$} & & & Down $^{*}(\mathrm{P}=0.003)$ \\
\hline
\end{tabular}

${ }^{a}$ Metabolites identified by standard comparison. ${ }^{b}$ Ionospheric models of mass spectrometry cationic scanning. ${ }^{\mathrm{c}}$ Comparison of characteristic metabolites integral peak area in the 3 groups. M/Z, mass-to-charge ratio; RT, retention time; LysoPC, lysophosphatidylcholine; MG, monoglyceride.
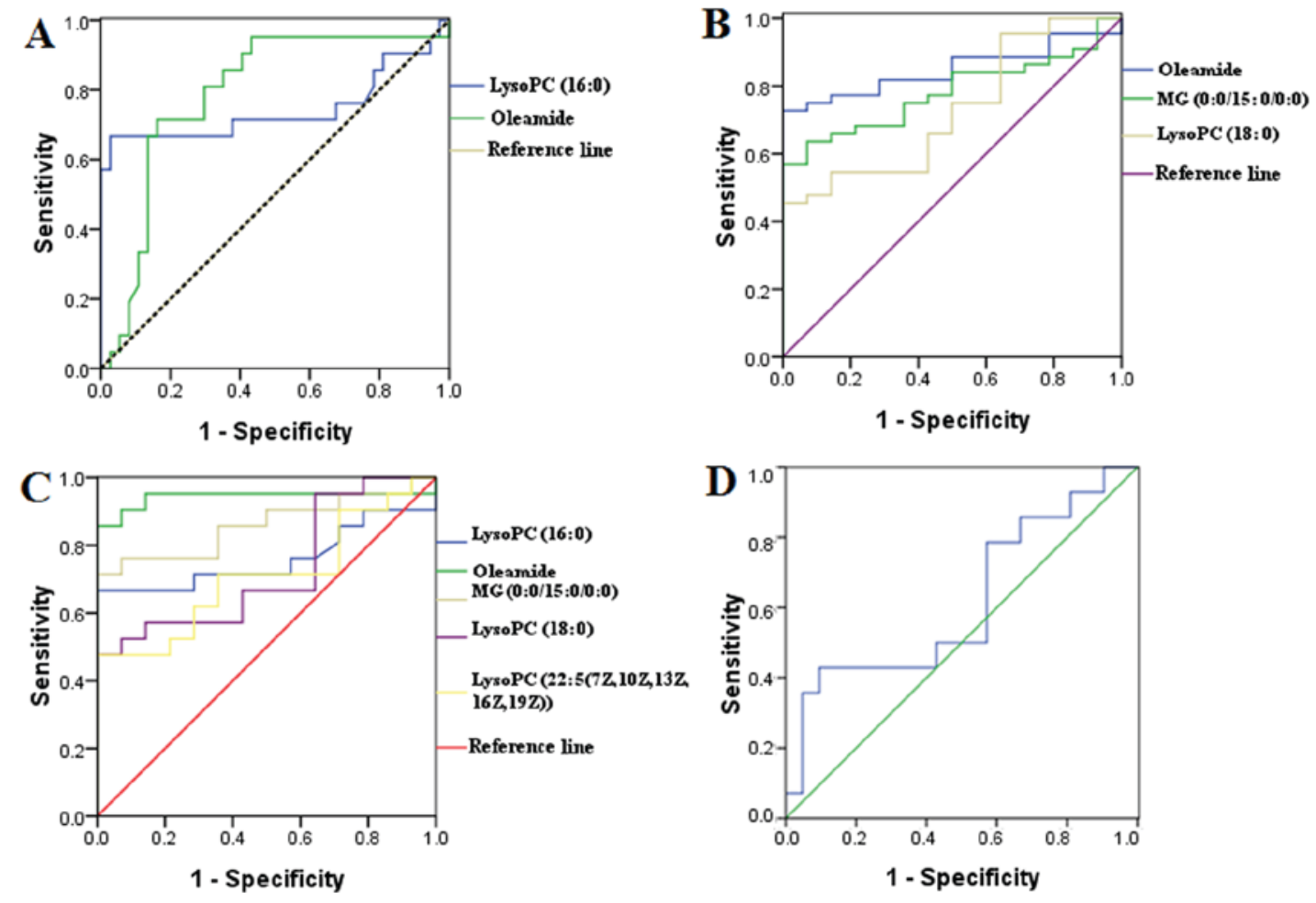

Figure 4. The receiver operator characteristic curve (ROC) of the identified metabolites and alpha-fetoprotein (AFP). (A) ROC of metabolites distinguishing group A from B and C; (B) ROC of metabolites distinguishing group C from A and B; (C) ROC of metabolites distinguishing groups A and C; (D) the AFP ROC distinguishing groups $\mathrm{A}$ and $\mathrm{C}$.

from all groups. No significant outliers were identified in the PCA model, indicating the pretreatment consistency was satisfactory and the analysis-detecting system was stable. However the PCA model did not show significant clustering tendency (Fig. 3A). In contrast, the OPLS-DA model (Fig. 3B), which constituted with the first predictive principal component and second predictive principal component, revealed a clustering tendency of data from each group.

Selection and identification of characteristic metabolites. For the purpose of exploring endogenous characteristic metabolites relating to differentiation grade, the characteristic metabolites affecting the clustering tendency of the OPLS-DA model within groups $\mathrm{A}, \mathrm{B}$ and $\mathrm{C}$ were selected following two steps: i) A new OPLS-DA model was established ( $\mathrm{R} 2 \mathrm{X}=66.2 \%, \mathrm{R} 2 \mathrm{Y}=91.1 \%, \mathrm{Q} 2=64.7 \%$ ) based on the three HCC groups (Fig. 3C); ii) Ions with a variable importance value $(\mathrm{VIP})>1$ were selected, and the ions which included zero in the confidence interval in the VIP diagram or the coefficient plot were excluded as previously described (17). Following these steps, 14 ions were selected as candidates for identification.

According to the process mentioned previously (18), 5 of the 14 selected ions were identified. The final results and statistical differences among the three HCC groups are presented in Table II. 
ROC analysis. The ROC curves were generated from the identified metabolites and alpha-fetoprotein (AFP; Fig. 4) to uncover the specificity of the previously identified metabolites in distinguishing HCC patients with diverse differentiation grades, and therefore their clinical value. The AUC of LysoPC (16:0) and Oleamide for distinguishing Group A from Groups $\mathrm{B}$ and $\mathrm{C}$ were 0.743 and 0.788 respectively. Moreover, the AUC of Oleamide, MG (0:0/15:0/0:0) and LysoPC (18:0) for distinguishing Group C from Groups A and B were 0.849, 0.781 and 0.727 respectively. In addition the AUC of LysoPC (16:0), Oleamide, monoglyceride (MG) (0:0/15:0/0:0), LysoPC (18:0) and LysoPC [22:5(7Z,10Z,13Z,16Z,19Z)] for distinguishing Group A from Group C were 0.760, 0.942, 0.861, 0.728 and 0.707 respectively. However, the AUC of AFP for distinguishing Group A from Group C was 0.616, with no significant difference.

\section{Discussion}

Identifying the correct differentiation grade of HCC is an important basis for the selection of an effective treatment that provides patients with the best long-term prognosis. DuBay et al (19) have previously reported that surgical resection of poorly differentiated HCC could increase patient mortality rates by 25 times. As reported previously, low-grade differentiation is an independent prognostic factor for HCC patients (20). Therefore, there is a clinical imperative to establish an effective strategy for identifying HCC patients with diverse differentiation grades. Histological methods such as biopsies are invasive and not generally accepted by patients. Imaging parameters, including contrast-enhanced ultrasound washout rate and apparent diffusion coefficient value, are related to HCC differentiation grades $(21,22)$, but remain unsatisfactory.

Metabolomics, a top-down systems biology approach, is often used to explore disease mechanisms and search for candidate diagnostic biomarkers. Therefore, the present study focused on the metabolic profile of HCC patients with diverse differentiation grades, with the aim of selecting characteristic metabolites to accurately identify each differentiation grade.

The five metabolites identified are lipid metabolites involved in inflammation and signal transduction, and it is well known that the liver is important in lipid metabolism. Unfortunately, the results could not explain why the amounts of metabolites differed among the three groups. To understand how these metabolites work in patients with different types of $\mathrm{HCC}$, further studies are required.

LysoPC is generated by the action of phospholipase A2 on membrane phosphatidylcholine, the most abundant cellular phospholipid (23). LysoPC has been reported to be decreased in patients with HCC (24). Compared with the control group, LysoPC [22:5(7Z,10Z,13Z,16Z,19Z)] was decreased in the HCC patients group (not shown). All three types of LysoPC demonstrated an ability to distinguish HCC patients with diverse differentiation grades. Decreased LysoPC levels may therefore indicate poorly differentiated HCC.

Oleamide is the prototype long chain primary fatty acid amide lipid messenger, first identified in human serum in 1989 (25). It is known to mediate the drive to sleep, has profound effects on thermoregulation, and acts as an analgesic in several models of experimental pain (25). The present study demonstrated a reduction in Oleamide levels when the differentiation degree decreased. Although its association with differentiation degree remains unclear, the ability of Oleamide to distinguish HCC patients with diverse differentiation merits further study.

In conclusion, the successful establishment of a metabolic profiling model revealed that utilizing metabolomics is a promising approach to find characteristic metabolites in serum that may be used to distinguish HCC patients with diverse differentiation grades. The 5 characteristic metabolites identified, Lysophosphatidylcholine (16:0), Oleamide, Monoglyceride (0:0/15:0/0:0), Lysophosphatidylcholine (18:0) and Lysophosphatidylcholine [22:5(7Z,10Z,13Z,16Z,19Z)], have this ability. Future research should focus on these characteristic metabolites to confirm their clinical value. In addition, research into the pathways related to these metabolites would provide reliable evidence supporting their use clinically.

\section{Acknowledgements}

The present study was supported by the Tianjin Research Program of Application Foundation and Advanced Technology (grant no. 13JCYBJC22100), Tianjin Research Program of Application Foundation and Advanced Technology (grant no. 16JCQNJC11600) and the General Item of Science and Technology Fund of Tianjin Municipal Bureau of Health (grant no. 2014KY01). The authors also thank Y. Wu (Clinical Laboratory Department Third Central Clinical College, Tianjin Medical University, Tianjin, China) for support.

\section{References}

1. El-Serag HB: Hepatocellular carcinoma. N Engl J Med 365: 1118-1127, 2011

2. Patel M, Shariff MI, Ladep NG, Thillainayagam AV, Thomas HC, Khan SA and Taylor-Robinson SD: Hepatocellular carcinoma: Diagnostics and screening. J Eval Clin Pract 18: 335-342, 2012.

3. Liu Y, Hong Z, Tan G, Dong X, Yang G, Zhao L, Chen X, Zhu Z, Lou Z, Qian B, et al: NMR and LC/MS-based global metabolomics to identify serum biomarkers differentiating hepatocellular carcinoma from liver cirrhosis. Int J Cancer 135: 658-668, 2014.

4. Qiang L, Huikai L, Butt K, Wang PP and Hao X: Factors associated with disease survival after surgical resection in Chinese patients with hepatocellular carcinoma. World J Surg 30: 439-445, 2006.

5. Shah SA, Greig PD, Gallinger S, Cattral MS, Dixon E, Kim RD, Taylor BR, Grant DR and Vollmer CM: Factors associated with early recurrence after resection for hepatocellular carcinoma and outcomes. J Am Coll Surg 202: 275-283, 2006.

6. Martins A, Cortez-Pinto H, Marques-Vidal P, Mendes N, Silva S, Fatela N, Glória H, Marinho R, Távora I, Ramalho F and de Moura MC: Treatment and prognostic factors in patients with hepatocellular carcinoma. Liver Int 26: 680-687, 2006.

7. Edmondson HA and Steiner PE: Primary carcinoma of the liver: A study of 100 cases among 48,900 necropsies. Cancer 7: 462-503, 1954.

8. Stigliano R, Marelli L, Yu D, Davies N, Patch D and Burroughs AK: Seeding following percutaneous diagnostic and therapeutic approaches for hepatocellular carcinoma. What is the risk and the outcome? Seeding risk for percutaneous approach of HCC. Cancer Treat Rev 33: 437-447, 2007.

9. Pawlik TM, Gleisner AL, Anders RA, Assumpcao L, Maley W and Choti MA: Preoperative assessment of hepatocellular carcinoma tumor grade using needle biopsy: Implications for transplant eligibility. Ann Surg 245: 435-442, 2007.

10. Nicholson JK, Lindon JC and Holmes E: 'Metabonomics': Understanding the metabolic responses of living systems to pathophysiological stimuli via multivariate statistical analysis of biological NMR spectroscopic data. Xenobiotica 29: 1181-1189, 1999. 
11. Fiehn O: Metabolomics-the link between genotypes and phenotypes. Plant Mol Biol 48: 155-171, 2002.

12. European Association of the Study of the Liver: 2011 European Association of the Study of the Liver hepatitis C virus clinical practice guidelines. Liver Int 32 (Suppl 1): 2-8, 2012.

13. de Bruijne J, Buster EH, Gelderblom HC, Brouwer JT, de Knegt RJ, van Erpecum KJ, Schalm SW, Bakker CM, Zaaijer HL, Janssen HL, et al: Treatment of chronic hepatitis $\mathrm{C}$ virus infection-Dutch national guidelines. Neth J Med 66: 311-322, 2008.

14. Pluskal T, Castillo S, Villar-Briones A and Oresic M: MZmine 2: Modular framework for processing, visualizing, and analyzing mass spectrometry-based molecular profile data. BMC Bioinformatics 11: 395, 2010.

15. Trygg J, Holmes E and Lundstedt T: Chemometrics in metabonomics. J Proteome Res 6: 469-479, 2007.

16. Eriksson L, Johansson E, Lindgren F, Sjostrom M and Wold S Megavariate analysis of hierarchical QSAR data. J Comput Aided Mol Des 16: 711-726, 2002.

17. Yin P, Wan D, Zhao C, Chen J, Zhao X, Wang W, Lu X, Yang S, $\mathrm{Gu} \mathrm{J}$ and $\mathrm{Xu} \mathrm{G}$ : A metabonomic study of hepatitis B-induced liver cirrhosis and hepatocellular carcinoma by using RP-LC and HILIC coupled with mass spectrometry. Mol Biosyst 5: 868-876, 2009.

18. Liu SY, Zhang RL, Kang H, Fan ZJ and Du Z: Human liver tissue metabolic profiling research on hepatitis B virus-related hepatocellular carcinoma. World J Gastroenterol 19: 3423-3432, 2013.
19. DuBay D, Sandroussi C, Sandhu L, Cleary S, Guba M, Cattral MS, McGilvray I, Ghanekar A, Selzner M, Greig PD and Grant DR: Liver transplantation for advanced hepatocellular carcinoma using poor tumor differentiation on biopsy as an exclusion criterion. Ann Surg 253: 166-172, 2011.

20. Yaprak O, Akyildiz M, Dayangac M, Demirbas BT, Guler N, Dogusoy GB, Yuzer Y and Tokat Y: AFP level and histologic differentiation predict the survival of patients with liver transplantation for hepatocellular carcinoma. Hepatobiliary Pancreat Dis Int 11: 256-261, 2012.

21. Jiang T, Wang J, Luo Z, Zhu K, Chen J and Shan H: Study of apparent diffusion coefficient value and histopathological differentiation of hepatocellular carcinoma. Zhonghua Yi Xue Za Zhi 95: 187-191, 2015 (In Chinese)

22. Feng Y, Qin XC, Luo Y, Li YZ and Zhou X: Efficacy of contrast-enhanced ultrasound washout rate in predicting hepatocellular carcinoma differentiation. Ultrasound Med Biol 41: $1553-1560,2015$

23. Ryborg AK, Johansen C, Iversen L and Kragballe K: Lysophosphatidylcholine induces keratinocyte differentiation and upregulation of AP-1- and NF-kappaB DNA-binding activity. Acta Derm Venereol 84: 433-438, 2004.

24. Zhou L, Ding L, Yin P, Lu X, Wang X, Niu J, Gao P and Xu G: Serum metabolic profiling study of hepatocellular carcinoma infected with hepatitis B or hepatitis $C$ virus by using liquid chromatography-mass spectrometry. J Proteome Res 11: 5433-5442, 2012.

25. Mueller GP and Driscoll WJ: Biosynthesis of oleamide. Vitam Horm 81: 55-78, 2009. 